\title{
Invited Editorial: “Enhanced Recovery Minimizes Opioid Use and Length of Stay in Patients Undergoing Mastectomy with Reconstruction"
}

\author{
Sarah M. DeSnyder, MD ${ }^{1}$, and Matthew M. Hanasono, MD $^{2}$ \\ ${ }^{1}$ Department of Breast Surgical Oncology, The University of Texas MD Anderson Cancer Center, Houston, TX; \\ ${ }^{2}$ Department of Plastic Surgery, The University of Texas MD Anderson Cancer Center, Houston, TX
}

Enhanced recovery after surgery (ERAS) protocols are multimodal perioperative interventions, centered around preoperative counseling, standardized analgesic and anesthetic regimens, and early mobilization, implemented with the goal of achieving faster recovery following surgery while simultaneously reducing costs. ${ }^{1}$ In their study, McGugin and colleagues compared intravenous opioid use and inpatient hospital length of stay (LOS) between subjects who underwent unilateral or bilateral mastectomy with breast implant or tissue expander (TE) reconstruction after implementation of an ERAS protocol, and historical controls who were treated prior to implementing the ERAS protocol. ${ }^{2}$ The authors found that regardless of laterality, mastectomy type, concomitant axillary lymph node or sentinel lymph node dissection, and prosthesis type (implant or TE), the subject group used less intravenous opioids for a shorter duration of time and had a shorter hospital LOS. Based on the results presented, this retrospective study makes a case for implementation of ERAS following mastectomy and implant-based breast reconstruction, which continues to be the most common method of breast reconstruction in the US, although several confounders are present that warrant further study.

The subjects notably had more patients who underwent reconstruction with prepectoral implant or TE placement. One perceived advantage of prepectoral placement is

(C) Society of Surgical Oncology 2019

First Received: 26 July 2019;

Published Online: 12 August 2019

M. M. Hanasono, MD

e-mail: mhanasono@mdanderson.org decreased pain, since the pectoralis major muscle is neither elevated from the chest wall nor is it stretched by an implant or TE. ${ }^{3}$ Acellular dermal matrices are used as a barrier, rather than the pectoralis major muscle, to decrease the rate of wound dehiscence and implant exposure. This pain-reducing technique was not utilized in the control group since the technique has only become popular in recent years. However, the rate of intravenous and oral opioid use for subjects who underwent prepectoral implant or TE placement was similar to those subjects who had more traditional subpectoral placement, arguing against this difference between subjects and controls being an important confounding variable.

One difference that was not recorded was whether patients in the subject group had a higher rate of partial muscular coverage compared with those in the control group. In recent years, the majority of reconstructive surgeons have taken to placement of the superior portion of the implant or TE beneath the pectoralis major muscle, and have covered the inferior portion of the prosthesis with acellular dermal matrix. In contrast, it was previously more common to elevate both the pectoralis major and the serratus anterior muscles to achieve total muscular coverage of the prosthesis. There is some evidence that the newer technique is associated with less pain because the serratus anterior muscle is left undisturbed. ${ }^{4}$ This represents an important potential confounding variable.

Perhaps the greatest confounding factors in this study have to do with the use of historical controls. In the past decade or two, there has been increasing pressure on care providers to minimize hospital stays and facilitate timely discharge independent of the use of formal ERAS protocols. In our own institution, there was a shift approximately 14 years ago to routine discharge of all mastectomy and 
implant-based reconstruction patients on postoperative day 1 , with the majority of surgeries being performed in an ambulatory care setting equipped only for 23-h hospital stays. Currently, longer hospital stays are virtually unheard of unless there is a perioperative complication or a known major medical condition that requires prolonged monitoring.

The use of non-narcotic medications to achieve postoperative pain control has been demonstrated to be a critical component of strategies to reduce postoperative oral opioid use. A recent publication examining an ERAS pathway for patients undergoing breast-conservation surgery demonstrated successful elimination of postoperative narcotic prescriptions by employing the use of preoperative medications, including acetaminophen and gabapentin, local analgesia infiltration at surgery, and postoperative ibuprofen alternating with acetaminophen. ${ }^{5}$ In addition, the importance of preoperative patient counseling to include the recommendation to utilize non-narcotic medications such as ibuprofen and acetaminophen first, and emphasizing that these medications alone may be enough to control postoperative pain, has been demonstrated to be critical in reducing the need for postoperative opioids after mastectomy. ${ }^{6}$ A limitation of the present study is that data were not available to determine whether the subject group received less intravenous opioids not because they had adequate pain control with non-narcotic analgesics but because they were transitioned to oral opioids earlier than those in the control group. It is also important to note that this study did not contain any data about the use of postoperative oral opioids or non-narcotic analgesics for the controls.

Concurrent with trends toward early hospital discharge have been efforts to reduce opioid use, as well as reduce opioid prescriptions to prevent misuse, chronic use, and diversion. Caregivers at every level, physicians, trainees, mid-level providers, and nurses have been educated about the risks associated with excessive opioid prescribing.
Therefore, findings of decreased intravenous opioid use may in part be ascribed to bias against opioids, rather than improved pain control.

Finally, it should be kept in mind that in this study, intravenous opioid use is a surrogate for pain control, which was not specifically tracked as an outcome, nor was patient satisfaction. An important measure in contemporary studies seeking to decrease opioid prescriptions in patients undergoing breast surgery is the ability to achieve optimal pain control while decreasing narcotic use. In this era of increased focus on and transparency around patient satisfaction, we must be cognizant that if our efforts negatively impact patient satisfaction, the ultimate outcome may not prove to be acceptable.

\section{REFERENCES}

1. Temple-Oberle C, Shea-Budgell MA, Tan M, et al. Consensus review of optimal perioperative care in breast reconstruction: enhanced recovery after surgery (ERAS) Society recommendations. Plast Reconstr Surg 2017;139(5):1056e-1071e.

2. McGugin CJ, Coopey SB, Smith BL, et al. Enhanced recovery minimizes opioid use and hospital stay for patients undergoing mastectomy with reconstruction. Ann Surg Oncol. 2019. https://d oi.org/10.1245/s10434-019-07710-3.

3. Tasoulis MK, Iqbal FM, Cawthorn S, et al. Subcutaneous implant breast reconstruction: time to reconsider? Eur J Surg Oncol 2017;43(9):1636-1646.

4. McCarthy CM, Lee CN, Halvorson EG, et al. The use of acellular dermal matrices in two-stage expander/implant reconstruction: a multicenter blinded, randomized controlled trial. Plast Reconstr Surg 2012;130(5 Suppl 2):57S-66S.

5. Rojas KE, Manasseh DM, Flom PL, et al. A pilot study of breast surgery Enhanced Recovery After Surgery (ERAS) protocol to eliminate narcotic prescription at discharge. Breast Cancer Res Treat 2018;171(3):621-626.

6. Lee JS, Howard RA, Klueh MP, et al. The impact of education and prescribing guidelines on opioid prescribing for breast and melanoma procedures. Ann Surg Oncol 2019;26(1):17-24.

Publisher's Note Springer Nature remains neutral with regard to jurisdictional claims in published maps and institutional affiliations. 